University of Wollongong

Research Online

Faculty of Engineering and Information

Faculty of Engineering and Information

Sciences - Papers: Part B

Sciences

2018

The effect of a dissipative ladle shroud on mixing in Tundish: Mathematical and experimental modelling

Jiangshan Zhang

University of Wollongong, jz822@uowmail.edu.au

Shufeng Yang

University of Science And Technology Beijing

Jingshe Li

University of Science And Technology Beijing

Haiyan Tang

University of Science And Technology Beijing

Zhengyi Jiang

University of Wollongong, jiang@uow.edu.au

Follow this and additional works at: https://ro.uow.edu.au/eispapers1

Part of the Engineering Commons, and the Science and Technology Studies Commons

Research Online is the open access institutional repository for the University of Wollongong. For further information contact the UOW Library: research-pubs@uow.edu.au 


\title{
The effect of a dissipative ladle shroud on mixing in Tundish: Mathematical and experimental modelling
}

\author{
Abstract \\ The effect of a dissipative ladle shroud (DLS) on mixing in tundish was investigated, compared with that \\ of a conventional ladle shroud (CLS) using mathematical and physical modelling. The tracer profiles of \\ mathematical results, achieved using large eddy simulation, were validated by physical observations \\ employing high-speed cinephotography. The design of a DLS dramatically changed the flow patterns and \\ contributed the intermixing of fluid elements inside the ladle shroud. The vortex flow encouraged the \\ turbulent mixing and was verified by tracking of physical tracer dispersion inside the DLS. Residence Time \\ Distribution (RTD) curves were obtained in two different sized tundishes to examine the mixing \\ behaviours. The findings indicated that the DLS benefited the tundish mixing in terms of increasing active \\ volume. The effect seemed to be more remarkable in the smaller tundish. The DLS gave rise to a more \\ plug-like flow pattern inside the tundish, showing potential to shorten the transition length during grade \\ change.

\section{Disciplines} \\ Engineering | Science and Technology Studies

\section{Publication Details} \\ Zhang, J., Yang, S., Li, J., Tang, H. \& Jiang, Z. (2018). The effect of a dissipative ladle shroud on mixing in \\ Tundish: Mathematical and experimental modelling. High Temperature Materials and Processes, 37 (1), \\ 25-32.
}


Jiangshan Zhang, Shufeng Yang, Jingshe Li, Haiyan Tang* and Zhengyi Jiang*

\section{The Effect of a Dissipative Ladle Shroud on Mixing in Tundish: Mathematical and Experimental Modelling}

DOI 10.1515/htmp-2016-0093

Received May 14, 2016; accepted January 12, 2017

\begin{abstract}
The effect of a dissipative ladle shroud (DLS) on mixing in tundish was investigated, compared with that of a conventional ladle shroud (CLS) using mathematical and physical modelling. The tracer profiles of mathematical results, achieved using large eddy simulation, were validated by physical observations employing high-speed cinephotography. The design of a DLS dramatically changed the flow patterns and contributed the intermixing of fluid elements inside the ladle shroud. The vortex flow encouraged the turbulent mixing and was verified by tracking of physical tracer dispersion inside the DLS. Residence Time Distribution (RTD) curves were obtained in two different sized tundishes to examine the mixing behaviours. The findings indicated that the DLS benefited the tundish mixing in terms of increasing active volume. The effect seemed to be more remarkable in the smaller tundish. The DLS gave rise to a more plug-like flow pattern inside the tundish, showing potential to shorten the transition length during grade change.
\end{abstract}

Keywords: large eddy simulation, ladle shroud, tundish, mixing, continuous casting

\footnotetext{
*Corresponding authors: Haiyan Tang, School of Metallurgical and Ecological Engineering, University of Science and Technology Beijing, Beijing 100083, P.R. China,

E-mail: tanghaiyan@ustb.metall.edu.cn

Zhengyi Jiang, School of Mechanical, Materials and Mechatronic Engineering, University of Wollongong, Wollongong NSW 2522, Australia, E-mail: jiang@uow.edu.au

Jiangshan Zhang, School of Metallurgical and Ecological Engineering, University of Science and Technology Beijing, Beijing 100083, P.R. China; School of Mechanical, Materials and Mechatronic Engineering, University of Wollongong, Wollongong NSW 2522, Australia

Shufeng Yang, Jingshe Li, School of Metallurgical and Ecological Engineering, University of Science and Technology Beijing, Beijing 100083, P.R. China
}

\section{Introduction}

At present, more than $95 \%$ of carbon steels and special steels rely on the process of continuous casting, in which several or tens of heats are cast continuously in one campaign [1]. Tundish is the last vessel before the solidification in the mould, thus quality of molten steel should be ensured with respect to composition, temperature and cleanness.

Mixing is an important function and task to homogenize molten steel in the liquid pool so as to improve the performance of the tundish. Poor mixing could result in big temperature difference between different zones (such as "dead zone") in a tundish and limit inclusion collision or floatation inside the tundish [1]. The mixing is dominated by the fluid flow, as the fluid motion generally falls into the scope of turbulence in the tundish. The performance of a tundish is largely affected by the flow characteristics of molten steel which thus have received a great deal of studies to optimize its operations [2-4]. There are basically two strategies to control the fluid flow and thus the mixing inside a tudnish: external forces and structure design of the tundish. The external forces can come from the incorporation of electromagnetic generators [5] or gas bubbling curtain $[6,7]$, which are able to control the flow pattern by imparting electromagnetic forces or extra buoyance forces. There are several ways to change the structure. Introducing flow control devices inside a tundish, such as turbulent inhibitor, baffle, impacting pad, weir and dam [1] to improve the performance of a tundish is very well known. The volume and depth of a tundish tend to be increased to minimize the turbulence in the tundish and avoid tundish slag carry-over to the mould [1]. Other parameters, such as curved-shape [8] and wall inclination [9] have been taken into account as well. Meanwhile there is a new concept to employ a ladle shroud, a tube connecting the ladle and the tundish, to perform flow control on the entry jet of the tundish for the purpose of controlling the fluid flow in the whole tundish without other devices. This method is estimated to be with the ease of simple operation and cost-effectiveness [10]. Trumpetshaped ladle shrouds were found to be successful in 
improving the mixing and benefiting the industrial practices [11]. Solorio-Diaz et al. [10] developed a swirling ladle shroud, which showed merits over conventional ladle shrouds (CLS) in terms of fluid flow and flotation of inclusions through buoyancy, drag and inertial forces. A novel ladle shroud, dissipative ladle shroud (DLS), was proposed and it is capable of dissipating turbulent energy inside the shroud and minimizing the whole turbulence inside the tundish [12]. The DLS was also found to be quite favourable by reducing the surface turbulence of slag-steel interface during ladle change-over periods [13], which makes this new ladle shroud draw increasingly attention. However, there is still no sufficient knowledge about the effect of DLS on mixing in the tundish. The lower part of the ladle shroud is considered, but mostly simplified as a constant inlet velocity profile in tundish simulation. The flow characteristics inside the shroud have not been paid sufficient attention. Simultaneously, the most popular Reynold Average Navier-Stokes (such as k- $-\varepsilon$ ) modelling shows limitation to study the flow and mixing inside the ladle shroud, due to its poor ability to capture small-scale eddies.

The purpose of the current study is to comparatively investigate the mixing behaviour inside a DLS and a CLS from the perspectives of flow characteristics and tracer transfer. Large eddy simulation (LES) is employed to have a detailed insight into the turbulent flow inside the ladle shroud. A technique of stimulus-response injection of a tracer is used to analyse the achieved Residence Time Distribution (RTD) curves and evaluate the mixing inside two different scaled tundishes.

\section{Experimental}

\section{Geometry}

\section{Ladle shrouds and a small tundish}

Models with a size of one-third scale of the real CLS and DLS were used for the physical and mathematical modelling. A schematic drawing of the two ladle shrouds and their dimensions are shown in Figure 1. The tube diameter of the CLS is $30 \mathrm{~mm}$. The diameters of the inlet and the outlet are respectively $30 \mathrm{~mm}$ and $60 \mathrm{~mm}$ for the DLS (Figure 1(a)). There are two tundishes that are studied for modelling. A small tundish, like a water tank, is chosen to carry out the LES simulation, due to the challenges to solve a full-scaled tundish with fine grid blocks and appropriate Yplus values to achieve accurate results.

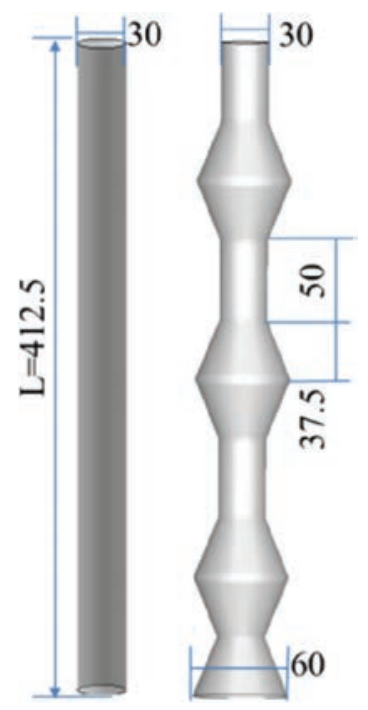

(a)

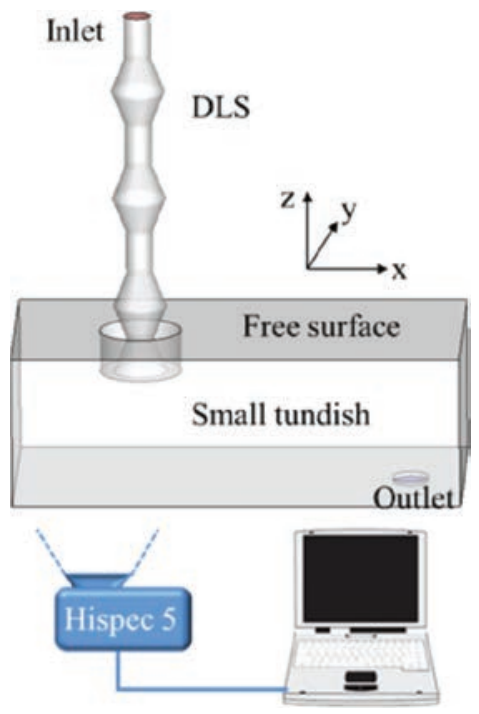

(b)
Figure 1: (a) Schemes and dimensions of studied ladle shrouds (the unit is $\mathrm{mm}$ for the listed values). (b) Experimental set-up and boundary conditions of the small tundish.

The other one is a one-third scale of a real tundish, which will be described in the following section. The length, width and height of the small tundish model are chosen to be $500 \mathrm{~mm}, 250 \mathrm{~mm}$ and $170 \mathrm{~mm}$ respectively. The computational domain is meshed into $\sim 1,750,000$ hexahedral cells.

For the boundary conditions, a constant inflow velocity $(0.708 \mathrm{~m} / \mathrm{s}$, derived from the inlet area and flow rate of $30 \mathrm{~L} / \mathrm{min}$ ) profile was applied at the nozzle inlet; the outlet was set with a fixed pressure of $0 \mathrm{~Pa}$ to the ambient. The top free surface was simplified as free-slip boundaries with zero normal velocity. Walls were considered to be non-slip, and the near wall boundary domain was handled using the Werner-Wengle formulations [14].

\section{A one-third scale tundish}

To further the study of the effect of ladle shrouds on mixing in a tundish. A one-third scale tundish, consistent with the size scale of the ladle shrouds, was built based on industrial practices. The dimensions and structure of the tundish model are shown in Figure 2. The tundish is used in a Chinese steelmaking plant to produce slabs with a cross section of $220 \mathrm{~mm} \times 2000 \mathrm{~mm}$. In water modelling, geometrical and dynamic Similarity criteria between the model and the prototype tundish were maintained, and the calculation procedure can be found in reference [15]. 


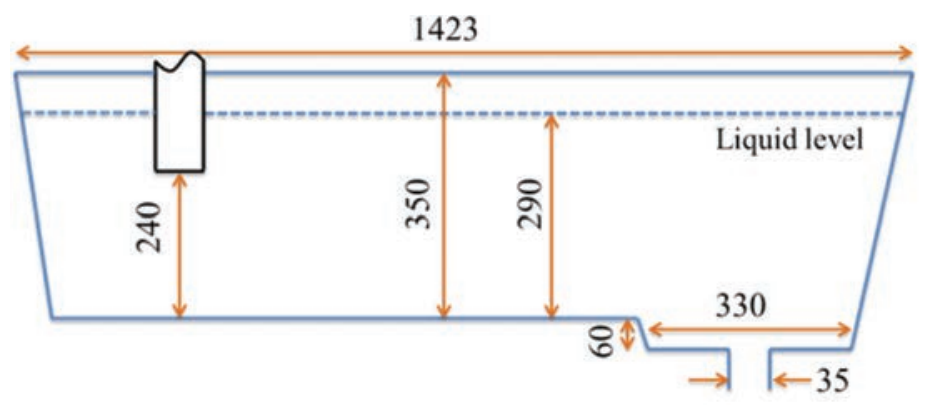

\section{Mathematical modelling}

Large eddy simulation (LES) is believed to be a more accurate and efficient method to get detailed information of turbulence compared with Reynolds-averaged approach and Direct Numerical Simulation [11]. LES adopts the concept of filtering and eddies are divided into large scale and sub-grid scale (SGS) categories. The filtering operation is offered by a finite-volume discretization in ANSYS-FLUENT as [16]:

$$
\bar{\varphi}(x)=\frac{1}{V} \int V \varphi\left(x^{\prime}\right) G\left(x, x^{\prime}\right) d x^{\prime}, x^{\prime} \in V
$$

where $\mathrm{V}$ represents the volume of the computational cell, and $G\left(x, x^{\prime}\right)$ is the filtering function. The filtered variables are denoted with an overbar. The filtered Navier-Stokes equations for incompressible fluids are as follows:

$$
\begin{gathered}
\frac{\partial \bar{u}_{i}}{\partial x_{i}}=0 \\
\frac{\partial \bar{u}_{i}}{\partial t}+\frac{\partial\left(\bar{u}_{i} \bar{u}_{j}\right)}{\partial x_{j}}=-\frac{1}{\rho} \frac{\partial \bar{p}}{\partial x_{i}}+\frac{\partial}{\partial x_{j}}\left(v_{0}+v_{t}\right)\left(\frac{\partial \bar{u}_{i}}{\partial x_{j}}+\frac{\partial \bar{u}_{j}}{\partial x_{i}}\right)-\frac{\partial \tau_{i j}}{\partial x_{j}}
\end{gathered}
$$

where $\rho, t, p$ and $u_{i}$ represent density, time, pressure and filtered velocities respectively. $v_{0}$ and $v_{t}$ are the molecule viscosity and eddy-viscosity. The subscripts $i$ and $j$ are the three directions in Cartesian coordinates. $\tau_{i j}$ represents the unknown SGS stresses:

$$
\tau_{i j}=\frac{1}{3} \vartheta_{i j} \tau_{k k}+2 v_{t} \bar{S}_{i j}
$$

where $\vartheta_{i j}$ represents the stress tensor resulted from molecular viscosity; the isotropic part of the SGS stresses $\tau_{k k}$ is added to the filtered static pressure term or neglected for incompressible flows [16]. $\bar{S}_{i j}$ indicates the rate of strain tensor

The Wall-Adapting Local Eddy-Viscosity (WALE) model is employed to address the SGS stresses, due to its ease of dynamic procedure and suitability to complicate geometries [17]. The $v_{t}$ is expressed as:

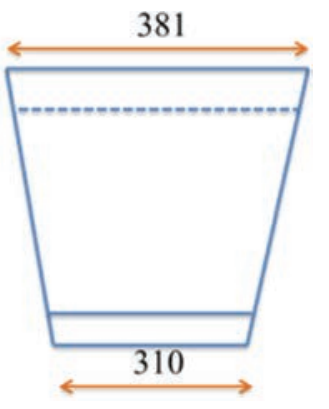

Figure 2: Schemes and dimensions of a one-third tundish. where $L_{s}$ is the mixing length for sub-grid scales, defined as below:

$$
L_{s}=\min \left(\kappa d, C_{w} V^{1 / 3}\right)
$$

where $d$ is the distance from the cell centre to the closest wall; the von Kármán constant $\kappa$ is $0.418 . C_{w}$ is chosen to be 0.325 , which has been confirmed to yield satisfactory results for a wide range of flow [16].

An instantaneous tracer transfer associated with fluid flow was modelled to track the tracer dispersion by solving the following equations:

$$
\frac{\partial C}{\partial t}+u \frac{\partial C}{\partial x}+v \frac{\partial C}{\partial y}+w \frac{\partial C}{\partial z}=D_{0}+\frac{v_{t}}{\rho S c_{t}}\left(\frac{\partial^{2} C}{\partial x^{2}}+\frac{\partial^{2} C}{\partial y^{2}}+\frac{\partial^{2} C}{\partial z^{2}}\right)
$$

where $D_{0}$ is the molecular diffusivity of a tracer; as turbulent flow dominates the mass transfer, the Schmidt number $\mathrm{Sc}_{\mathrm{t}}$ was chosen as one. The solutions of the species transfer and LES turbulent model are coupled together to acquire an unsteady mass distribution of the tracer.

\section{Physical modelling and validation}

A water model was established to validate the LES modelling with the same flow parameters as the small tundish. The fluid flows inside the DLS and the tundish has been verified in the previous study [11]. For the mixing, the movement of a tracer by injecting black ink from the inlet in the physical water model was recorded using a high-speed camera Hispec 5 (Figure 1(b)). The transient concentration profiles of the tracer at different times were filmed and are shown in Figure 3(a)-3(d). Correspondingly, tracer profiles of the LES are shown in Figure 3(e)-3(h). At around 0.100 s (after the injection of the tracer), the ink flows towards the left side of the first chamber (Figure 3(a) and 3(e)), and travels down 


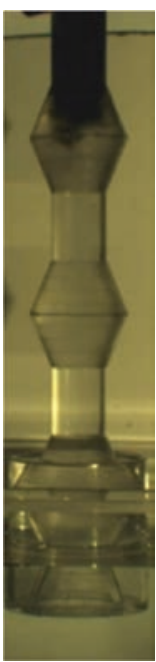

(a)

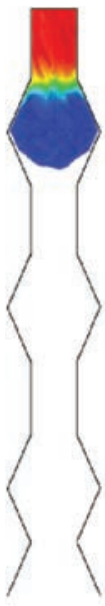

(e)

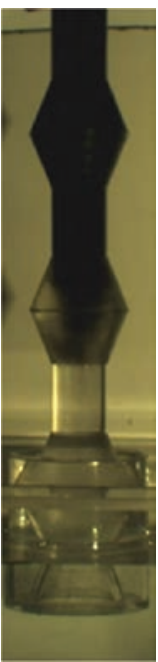

(b)

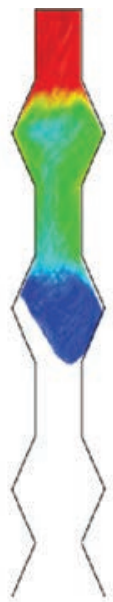

(f)

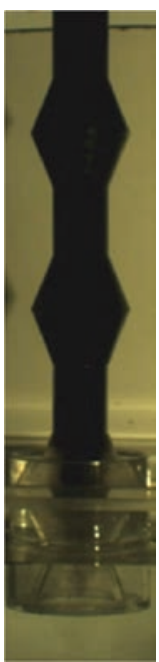

(c)

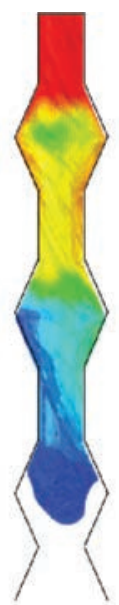

(g)

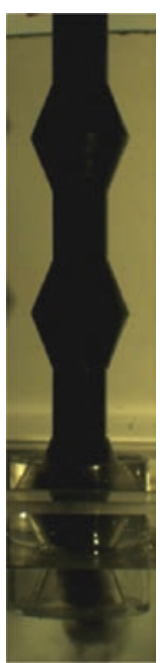

(d)

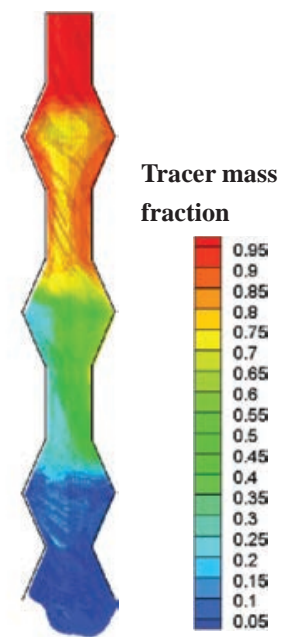

(h)
Figure 3: Tracer dispersion profiles at different time: (a) $0.104 \mathrm{~s}$, (b) $0.304 \mathrm{~s}$, (c) $0.477 \mathrm{~s}$, and (d) $0.598 \mathrm{~s}$ in the physical modelling; (e) $0.100 \mathrm{~s}$, (f) $0.300 \mathrm{~s},(\mathrm{~g}) 0.470 \mathrm{~s}$ and (h) $0.610 \mathrm{~s}$ in the LES modelling.

along the right-side wall of the second chamber at about 0.300 s (Figure 3(b) and 3(f)). The pioneer tracer finally leaves out the shroud at about $0.600 \mathrm{~s}$ and tends to flow towards the right side (Figure 3(d) and 3(h)). The results of the two models match well with respect to the dispersion profiles at different time.

Stimulus-response experiments were conducted using the tracer to obtain RTD curves by injecting $50 \mathrm{ml}$ saturated $\mathrm{KCl}$ solution from the inlet in the one-third scale tundish. The electric conductivity was recorded at the outlet with an interval of $1.0 \mathrm{~s}$. The characterization of the dead volume, plug volume and mixed volume have been well developed for single-strand tundishes in reference [18].

\section{Results and discussion}

\section{Flow and mixing inside the ladle shrouds}

The mass transfer is dominated by the fluid flow inside the ladle shroud with high turbulence (Reynolds number, $\sim 20,000$ ). It is reasonable to elucidate mixing from the point view of fluid flow. Vortex (or eddy) is one of the typical attributes of turbulent flow, which implies the rotation of fluid elements as they travel in a fluid field. A measurement of rotation, vorticity, is defined as the curl of the velocity vector $\left(\zeta=\nabla \times u_{i}\right)$. The interaction of different scale eddies, dissipation in particular, strongly contributes the intermixing of fluid elements [19], which can be characterized by the rate of turbulence energy dissipation $(\varepsilon)$. The relationship between the vorticity and the dissipation rate is illustrated as [11]:

$$
\varepsilon=v_{t} \overline{\zeta_{i} \zeta_{i}}
$$

Thus, the existence and magnitude of vortices, along with the basic notion of flow velocity, are selected as the main features of turbulence to facilitate the discussion of mixing inside the ladle shrouds in the succeeding sections.

A typical comparison of the flow profiles inside the CLS and the DLS is shown in Figure 4. The directions of velocity vectors in the CLS seem to be consistent and flow straight down along the ladle shroud (Figure 4(a)), similar to the plug flow. Smaller velocity vectors are observed along the wall boundaries and virtually no vortex can be

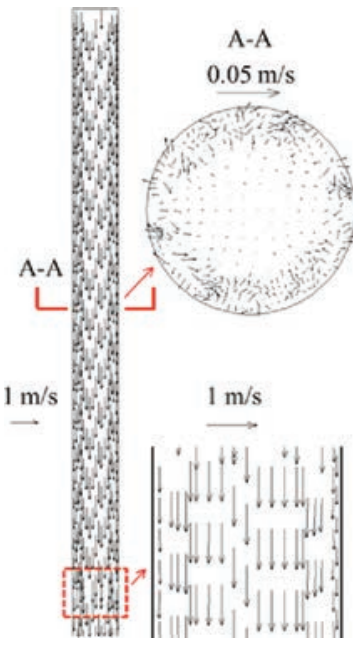

(a)

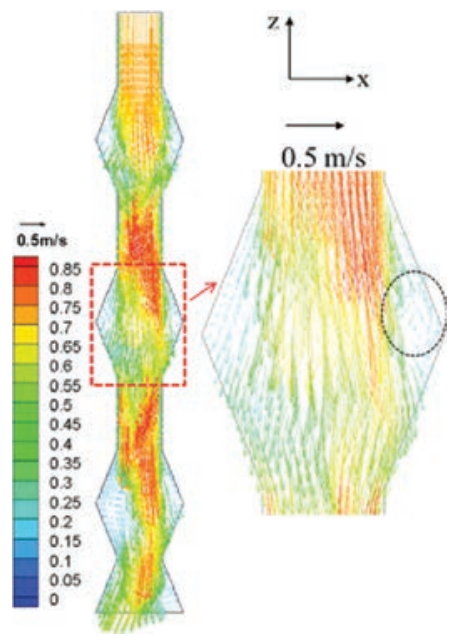

(b)
Figure 4: Typical velocity vector profiles, with enlarged transversal and longitudinal section of the (a) CLS and (b) DLS at the central XZ plane. 
visualized on the longitudinal (XZ) plane in the enlarged view. On the transversal plane (XY), the velocity is quite small in the $\mathrm{X}$ and $\mathrm{Y}$ directions, less than $0.03 \mathrm{~m} / \mathrm{s}$. Only weak vortices are created along the wall due to the boundary effect. In the DLS, the velocity decreases in the chambers and increases in the straight sections; many vortices are formed inside the shroud, especially inside the chambers. The main stream swings inside the shroud, and vortices are intensified at the enlarged sections of the chamber in Figure 4(b). Meanwhile, an anticlockwise vortex is formed at the corner of the chamber, which can drive the intermixing of neighbour fluid elements in longitudinal direction.

To clearly reveal the fluid flow in the DLS, velocity vector and contour profiles are also displayed in the transversal sections of the three chambers and the outlet in Figure 5. The colour indicates the overall velocity magnitude in the XYZ directions; the arrow is just the velocity vector in the $\mathrm{XY}$ plane. Tangential velocity vectors can be observed and numerous vortices are formed on the selected planes which are mainly caused by the sudden change of the velocity direction from the divergent part into the convergent part of the chamber. A variety of time-dependent vortices is formed and can intermix with the longitudinal vortices in Figure 4(b). The velocity in XY plane reaches nearly $0.2 \mathrm{~m} / \mathrm{s}$, which is much higher than that of CLS. This means the mass transfer is radially intensified inside the DLS. The vortices observed in the selected sections imply that the fluid elements are easy to intermix with each other both in the longitudinal and radial directions inside the DLS. This can also be verified by the ink dispersion profiles filmed by a high-speed camera in Figure 6. At $0.1728 \mathrm{~s}$ (after the injection of ink in another tracer test), the stream flows down mainly towards the left side and in prone to turn anticlockwise to the right side. At $0.2065 \mathrm{~s}$ and $0.2445 \mathrm{~s}$, the anticlockwise vortex is fully developed.

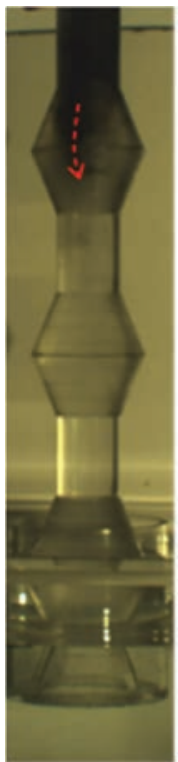

$0.1728 \mathrm{~s}$

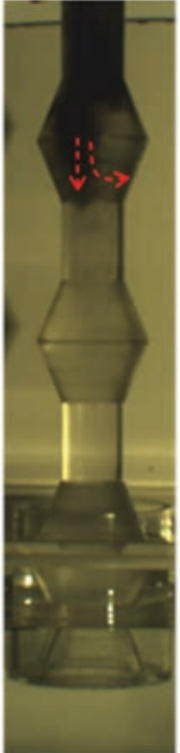

$0.2065 \mathrm{~s}$

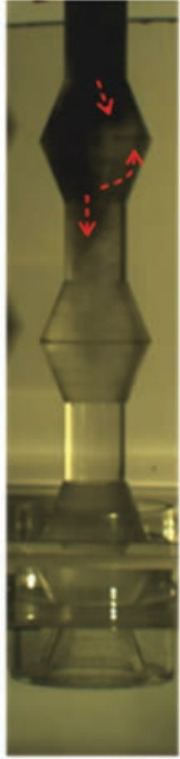

$0.2445 \mathrm{~s}$

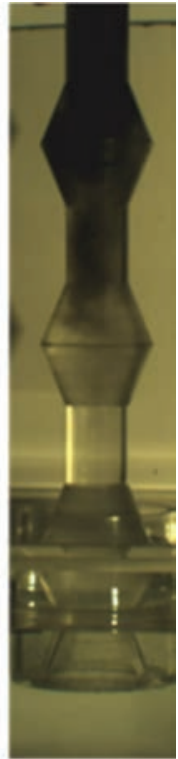

$0.2892 \mathrm{~s}$
Figure 6: Ink dispersion in the first chamber filmed by a high-speed camera.

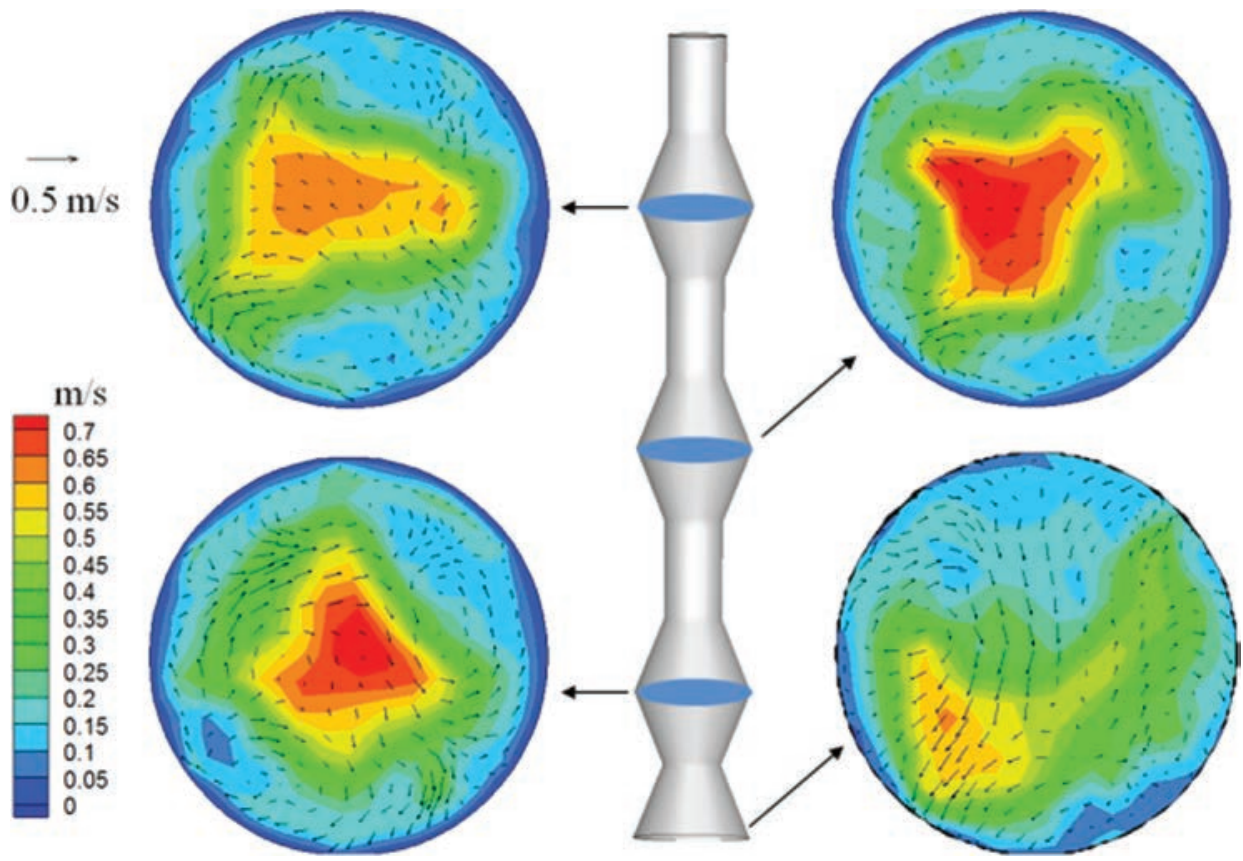

Figure 5: Velocity vector and contour profiles in the transversal sections of the three chambers and the outlet. 
With the effect of the flow and diffusion, the ink finally gets mixed inside the first chamber.

The above discussion indicates that the design of three chambers has revolutionized the flow structure and mixing inside the ladle shroud. The reinforced turbulence enhances the movement and intermixing of fluid elements in all directions, rather than solely flowing straight down with less interaction. In other words, the fluid premixing ahead of the tundish is improved inside the ladle shroud, being potentially changing the mixing inside the tundish as well.

\section{Mixing in Tundishes}

Stimulus-respond technique was utilized to examine the mixing phenomena by analysing RTD curves. The analysis of RTD characteristics is deemed as an effective approach to evaluate the plug volume, dead volume and mixed volume. The comparison in different scaled tundishes could be helpful to investigate the effect of ladle shroud on different sized tundishes as well as the inner and outer outlets in multi-strand tundishes.

\section{In the small tundish}

The RTD curve profiles of the studied cases are given in Figure 7 and the analysed results are shown in Table 1 for the small tundish. The time and concentration of tracer are both dimensionless to facilitate the discussion in Figure 7. It can be seen that the RTD curve of the DLS is much smoother than that of the CLS, with low

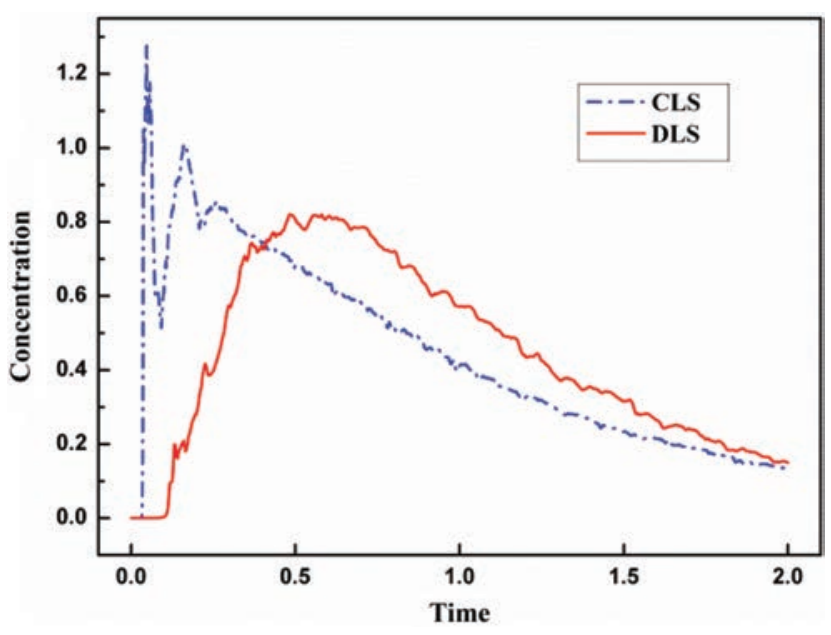

Figure 7: RTD curves of different ladle shrouds in the small tundish.
Table 1: RTD characteristics of studied cases in two types of tundishes.

\begin{tabular}{crrrrr}
\hline & & $\begin{array}{r}\text { Plug volume } \\
\mathbf{V}_{\mathbf{p}}(\%)\end{array}$ & $\begin{array}{r}\text { Dead volume } \\
\mathbf{V}_{\mathbf{d}}(\%)\end{array}$ & $\begin{array}{r}\mathbf{V}_{\mathbf{p}} / \\
\mathbf{V}_{\mathbf{d}}\end{array}$ & $\begin{array}{r}\text { Breakthrough } \\
\text { time (s) }\end{array}$ \\
\hline Small & CLS & 3.53 & 28.00 & 0.13 & 1.5 \\
tundish & DLS & 11.53 & 10.79 & 1.07 & 4.9 \\
$\begin{array}{c}\text { One-third } \\
\text { tundish }\end{array}$ & CLS & 16.47 & 23.78 & 0.69 & 41.5 \\
& DLS & 23.61 & 14.32 & 1.65 & 59.5 \\
\hline
\end{tabular}

peak concentration and relatively long breakthrough time. When the CLS is replaced by the DLS, the plug volume is dramatically increased from $3.53 \%$ to $11.53 \%$, and the dead volume is reduced from $28.00 \%$ to $10.79 \%$ respectively. A longer breakthrough time (1.5 $\mathrm{s}$ and $4.9 \mathrm{~s}$ for the CLS and DLS, respectively) is contributed. These are attributed to the swinging jet flow and damping effect of the DLS to mix with more fluid around the jet, rather than directly impacting the tundish bottom or quickly flowing out from the tundish outlet [12]. Although the outlet of the CLS is featured with higher velocity than that of the DLS, the tundish under the DLS is allowed with larger active volume (the sum of plug and mixing volume [18]). The high turbulent energy of the CLS jet is partially consumed by impacting on the tundish bottom and fluctuating the free surface, rather than by activating the fluid flow inside the tundish.

\section{In the one-third scale tundish}

The one-third scale tundish matches the size of the ladle shrouds, and it is expected to perform more closely to the real tundish. Meanwhile, all-scale turbulence information is included in the physical modelling; no time averaging or filtering is involved to simplify the fluid flow. Hence, physical modelling is considered to be a direct method to study the mixing in tundish [18]. The achieved RTD curves are shown in Figure 8. Microscopic fluctuation of the electric conductivity can be seen along the RTD curves. The breakthrough time is $59.5 \mathrm{~s}$ (in Table 1) with the DLS, longer than that with the CLS (41.5s), bringing about an increase of plug volume $14.67 \%$ to $23.61 \%$. Meanwhile, the values of $V_{p} / V_{d}$ are augmented with the DLS in the two tundishes. Thus, the dissipation effect of the DLS is effective in the two tundishes to benefit the flow characteristics, potentially improving cleanness and diminishing the differences of temperature and composition for the molten steel in practice. It is also noted that the increase of the $\mathrm{V}_{\mathrm{p}} /$ $V_{d}$ is $723 \%$ (from 0.13 to 1.07) in the small tundish, and is 


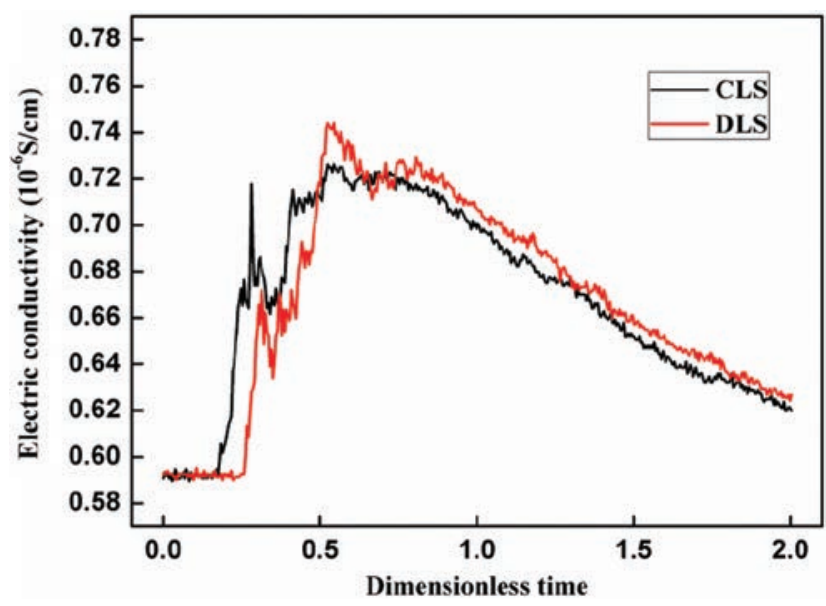

Figure 8: RTD curves of different ladle shrouds in the one-third scale tundish.

$139 \%$ (from 0.69 to 1.65 ) in the one-third scale tundish, when the CLS is replaced with the DLS. Thus, the DLS seems to perform more effectively in improving the fluid mixing in the smaller volume tundish than in the larger volume tundish due to its increased volume weight (volume of ladle shroud/ volume of tundish) to control the fluid flow. The comparison of mixing in these two single-strand tundishes with different sized can be also extended to the mixing in multi-strand tundishes, such as four or six-strand tundish. The outlets of the small and the one-third scaled tundishes correspond to the inner outlet and outer outlet (using the position of ladle shroud as reference) in a multi-strand tundish. The DLS can especially benefit the flow characteristics and mixing for the flow of the inner outlet (s), which is consistent to the study of Morales-Higa et al. [12].

In addition, grade transition frequently happens in a single casting sequence, which involves intermixing of the pre-existing steel and the newly introduced steel from the ladle shroud inside a tundish [20]. It is desirable to minimize the transition length (or tonnage) of hybrid chemistry product, as it commonly fails to meet the required composition of any grade. A perfect plug flow in a tundish would obtain an ideal steel grade transition without any intermixing. Fortuanately, the adoption of the DLS is able to contribute a larger plug volume (as just discussed above, and also applicable in transition operation), which means the DLS can promote a more plug-like flow pattern inside the tundish. In this case, a shorter transition length can be expected to reduce the tonnage of hybrid product.

It should be pointed out that the present study only deals with the fluid flow and mass transfer in reduced scale of ladle shrouds and tundish models. Water is used as fluid to replace molten steel to simplify the validation and discussion, which is a common alternative to study steelmaking practices. Future attention should be paid on having a more practical insight into the ladle shrouds.

\section{Conclusions}

Mathematical and physical modelling have been employed in the current study to investigate the mixing phenomenon inside the shrouds and two tundishes with the DLS and the CLS. Many vortices are observed and turbulence is reinforced inside the chambers, which result in intensified longitudinal and radial mass transfer and mixing inside the DLS. The DLS exerts dissipative effect on the jet flow of the ladle shroud and benefits the mixing in the tundish. The RTD analysis indicates that the DLS is effective to facilitate the flow characteristics and mixing both in the small and the one-third scale tundish, while being more effective in the small tundish. The DLS encourages the plug-like flow pattern inside the tundish, which is expected to reduce the transition product with hybrid composition during grade change.

Acknowledgements: Jiangshan Zhang would like to appreciate the financial support of Chinese Scholar Council (CSC).

Funding: The authors are indebted to the funding of National Science Foundation of China (No. 51304016) and Research and Development Funds of State Key Laboratory of Advanced Metallurgy, China (No.41603014).

\section{List of Symbols}

\author{
Greek Symbols \\ $\varphi \quad$ Any function \\ $\bar{\phi} \quad$ Filtered function \\ $\rho \quad$ Mass density $\left(\mathrm{kg} / \mathrm{m}^{3}\right)$ \\ $\tau_{i j} \quad$ Sub-grid Scale stress (N) \\ $\tau_{k k} \quad$ Isotropic part of the SGS stresses (N) \\ $\zeta_{i j} \quad$ Vorticity tensor \\ $\vartheta_{i j} \quad$ Stress tensor due to molecular viscosity \\ $v_{0} \quad$ Molecule viscosity $(\mathrm{kg} /(\mathrm{m} \bullet \mathrm{s}))$ \\ $v_{\mathrm{t}} \quad$ Turbulent eddy-viscosity $(\mathrm{kg} /(\mathrm{m} \bullet \mathrm{s}))$ \\ $\kappa \quad$ Von Kármán constant \\ Roman Symbols \\ $G \quad$ Filter function \\ $u_{i} \quad$ Velocity in direction “ $i$ ” (m/s)
}


$V \quad$ Volume of the computational cell $\left(\mathrm{m}^{3}\right)$

$t \quad$ Time (s)

$p \quad$ Pressure $(\mathrm{Pa})$

$S \quad$ Rate of strain tensor

$D_{0} \quad$ Molecular mass transfer coefficient $\left(\mathrm{m}^{2} / \mathrm{s}\right)$

$\mathrm{Sc}_{\mathrm{t}} \quad$ Schmidt number

$L_{s} \quad$ Mixing length for sub-grid scales (m)

$C_{w} \quad$ Constants in turbulence model

C Concentration of the tracer $(\mathrm{mol} / \mathrm{L})$

\section{References}

[1] Y. Sahai and T. Emi, Tundish Technology for Clean Steel Production, World Scientific Publishing Company, New Jersey, (2008).

[2] K. Mills, P. Ramirez-Lopez and P. Lee, High Temp. Mater. Processes, 31 (2012) 221-229.

[3] W. Caley, High Temp. Mater. Processes, 25 (2006) 157-166.

[4] S. Chatterjee and K. Chattopadhyay, Metall. Materi. Trans. B, 47 (2016) 508-521.

[5] J. Lavers and L. Kadar, Appl. Math. Model., 28 (2004) 29-45.

[6] L.C. Zhong, L.Y. Li, B. Wang, L. Zhang, L.X. Zhu and Q.F. Zhang, Ironmak. Steelmak., 35 (2008) 436-440.
[7] S. Chang, L. Zhong and Z. Zou, ISIJ Int., 55 (2015) 837-844.

[8] A. Tripathi and S.K. Ajmani, ISIJ Int., 45 (2005) 1616-1625.

[9] Y. He and Y. Sahai, Metall. Trans. B, 18 (1987) 81-92.

[10] G. Solorio-Díaz, R.D. Morales and A. Ramos-Banderas, Int. J. Heat Mass Transfer, 48 (2005) 3574-3590.

[11] J. Zhang, J. Li, Y. Yan, Z. Chen, S. Yang, J. Zhao and Z. Jiang, Metall. Materi. Trans. B, 47 (2015) 495-507.

[12] K. Morales-Higa, R. Guthrie, M. Isac and R.D. Morales, Metall. Mater. Trans. B, 44 (2013) 63-79.

[13] S. Garcia-Hernandez, R.D. Morales, J. de Jesus Barreto, I. Calderon-Ramos and E. Gutierrez, Steel Res. Int., 87 (2015) 1-14.

[14] U. Piomelli and E. Balaras, Annu. Rev. Fluid Mech., 34 (2002) 349-374.

[15] S. Yang, L. Zhang, J. Li and K. Peaslee, ISIJ Int., 49 (2009) 1551-1560.

[16] ANSYS Inc., FLUENT6.3-Manual. ANSYS Inc., Lebanon, NH (2007).

[17] F. Nicoud and F. Ducros, Flow Turbul. Combust., 62 (1999) 183-200.

[18] Y. Sahai and T. Emi, ISIJ Int., 36 (1996) 667-672.

[19] P.E. Dimotakis, J. Fluid Mech., 409 (2000) 69-98.

[20] X. Huang and B.G. Thomas, Metall. Mater. Trans. B, 27 (1996) 617-632. 